Gesnerus 53 (1996) 87-99

\title{
Tietze und die Zytomegalie-Erkrankung der Speicheldrüsen
}

Peter Diosi

\section{Summary}

Tietze has observed in 1898 the presence of peculiar huge cells in a tumor excized from the parotid gland of an infant. He held the structures for protozoan parasites immigrated from the oral cavity, but they turned out to be cytomegalic inclusion cells. Hence, the first description of cytomegaly-associated symptomatic illness dates back to Tietze's paper. It appears that he was the first author to establish the diagnosis in vivo, and his patient was the first one reported having survived this disease.

\section{Zusammenfassung}

Im Jahre 1898 hatte Tietze in den Drüsengängen einer von der Parotis eines Säuglings entfernten Geschwulst die Anwesenheit eigentümlicher grosser Zellen beobachtet. Er hielt diese Gebilde für aus der Mundhöhle eingewanderte Protozoen, doch der damals erstellte histologische Befund liess später klar erkennen, dass es sich um protozoenähnliche zytomegale Zellen handelte. Demnach ist die erste Beschreibung eines mit der Zytomegalie verbundenen Krankheitsbildes auf Tietze zurückzuführen. Der von ihm berichtete Fall war ferner der erste zu Lebzeiten eines Patienten erkannte Zytomegaliefall, und sein Patient war der erste Überlebende dieser Krankheit, von dem in der Literatur berichtet wurde.

Dr. Peter Diosi, Alteburger Strasse 298, D-50968 Köln 


\section{Einführung}

Das zuerst von Ribbert 1904 berichtete Vorkommen protozoenähnlicher Zellen im Epithelium der Speichelgänge der Parotis ${ }^{1}$ deutete diese Drüsen als häufigen Aufenthaltsort der Gebilde an. Sein Assistent Löwenstein, der daraufhin die pathologischen Wachstumsvorgänge an der kindlichen Parotis untersucht hatte, schrieb 1907: «Man kann schon jetzt behaupten, dass die Parotis der Hauptsitz der Gebilde ist $»^{2}$, er fand aber die Unterkieferspeicheldrüsen stets frei von den merkwürdigen Zellgebilden ${ }^{3}$. Wagner fand hinterher die protozoenartigen Zellen ebenfalls ausschliesslich in den Ausführungsgängen der Speicheldrüsen, jedoch «sowohl in der Glandula parotis wie Submaxillaris wie Sublingualis» ${ }^{4}$, und später konnte die Anwesenheit der seltsamen Gebilde im Drüsenepithel des Unterkiefers bei 12\% aller obduzierten Kinder festgestellt werden 5 . Dieses häufige Vorkommen führte dazu, dass die bis dahin weiter nicht beachteten Gebilde in ausgedehnterem Masse berücksichtigt wurden.

\section{Das Vorkommen bei Nagetieren und Menschen}

Gleichartige Zellen waren inzwischen auch in den Speicheldrüsen verschiedener Nager beobachtet und einer verborgenen Virusinfektion zugeschrieben worden ${ }^{6}$. Daher wurde das verursachende Agens auch beim Menschen «Speicheldrüsenvirus» (salivary virus oder salivary gland virus) genannt ${ }^{7}$ und als saprophytisches Virus betrachtet, das gewöhnlich keine erkennbaren klinischen oder pathologischen Veränderungen verursacht. Die merkwürdige Beschränkung der protozoenähnlichen Zellen auf die Speicheldrüsen wurde später einerseits auf das Stillen mit virusinfizierter Muttermilch zurückge-

1 Hugo Ribbert, «Ueber protozoenartige Zellen in der Niere eines syphilitischen Neugeborenen und in der Parotis von Kindern». Cbl. allg. Path. path. Anat. 15/23, 945-949 (1904).

2 Carl Löwenstein, «Ueber protozoënartige Gebilde in den Organen von Kindern». Cbl. allg. Path. path. Anat. 18/13, 513-518 (1907), S. 518.

3 Ibid., S. 518.

4 Hans Wagner, «Zur Kenntnis der sprotozoenartigen Zellen> in den Organen von Kindern». Beitr. path. Anat. 85, 145-164 (1930), S. 154.

5 Sidney Farber and S. Burt Wolbach, «Intranuclear and cytoplasmic inclusions («protozoanlike bodies») in the salivary glands and other organs of infants». Amer. J. Path. 8/2, 123-135 (1932), S. 128.

6 Rufus Cole and Ann G. Kuttner, «A filterable virus present in the submaxillary glands of guinea pigs». J. exp. Med. 44, 855-873 (1926).

7 C. H. Andrewes, «Immunity to the salivary virus of guinea-pigs studied in the living animal, and in tissue culture». Brit. J. exp. Path. 11, 23-34 (1930). 
führt $^{8}$, anderseits der relativen Unwirksamkeit der örtlichen Abwehrreaktionen zugeschrieben ${ }^{9}$. Vereinzelte Mitteilungen berichteten jedoch, dass das lokalisierte Vorkommen protozoenartiger Zellen in den Speicheldrüsen gelegentlich zu einem gutartigen lymphoepithelialen Tumor führe.

\section{Ribbert's Abhandlung}

1904 hat Ribbert in einem im Zentralblatt für Pathologie veröffentlichten Artikel (s. oben) mitgeteilt, dass er vor Jahren, als er die Parotis eines an einer nicht angegebenen Krankheit verstorbenen einjährigen Kindes untersuchte, in den sonst intakten Gängen der Drüse ungewöhnlich grosse Zellen angetroffen habe, die den Epithelien nur anlagen oder sich zwischen die Zellen hineinzudrängen schienen. Er fand sie teils einzeln, seltener gruppenweise. Einzelne Läppchen waren reichlich, andere gar nicht mit ihnen versehen, und im Bindegewebe fehlten sie ganz. Es fehlten ebenso jegliche Übergangsformen zwischen den grossen Zellen und den normalen Epithelien.

Bei einer weiteren Obduktion fand Ribbert in der Parotis eines Kleinkindes ein fast nur aus Gefässen bestehendes Gewebe, das dem eines Angioms entsprach und sich wie eine im Bereich der Parotis ausbreitende und die Gänge und Alveolen auseinanderdrängende Teleangiektasie verhielt. Auch in diesem Fall fand Ribbert die grossen Zellen ausschliesslich in das Lumen der Gänge eingelagert, welche sie auf kürzere oder längere Strecken völlig ausfüllten. Ihrer Erscheinung nach erinnerten die Gebilde an Amöben, doch Ribbert zögerte, sie als Protozoen einzustufen, denn «in der Niere (könnte es) den Anschein gewinnen, als seien sie aus ihren Bestandteilen abzuleiten» ${ }^{10}$. Als die konsultierten Zoologen Ehlers und Rhumbler diesbezüglich keine bestimmte Entscheidung treffen wollten, bezeichnete er die Gebilde als «protozoenartige» Zellen.

\section{Die Einwirkung des Berichts von Jesionek und Kiolemenoglu}

Ribbert hatte über die beobachteten Gebilde jahrelang nichts mitgeteilt, da ihm die Bedeutung der Befunde nicht ausreichend klar werden wollte. Erst

8 Peter Diosi, Livia Babusceac, Olimpia Nevinglovschi and Georg Kun-Stoicu, «Cytomegalovirus infection associated with pregnancy». Lancet 1063-1066 (1967/2), S. 1066.

9 Karl Benirschke, G. R. Mendoza, and P. L. Bazeley, «Placental and fetal manifestation of Cytomegalovirus infection». Virchows Arch. (Cell. Path.) 16/2, 121-139 (1974).

10 Ribbert (No. 1), S. 946. 
das Erscheinen im Jahre 1904 des Berichts der Münchener Dermatologen Albert Jesionek und Basilius Kiolemenoglu über die Anwesenheit ähnlicher Gebilde in den inneren Organen eines totgeborenen luetischen Fötus ${ }^{11}$ ermutigte ihn zur Veröffentlichung seiner eigenen Beobachtungen: «Die Abhandlung von Jesionek und Kiolemenoglu veranlasst mich, (...) auch mit meinen Erfahrungen nicht zurückzuhalten» ${ }^{12}$. Obwohl er vorher von «hypertrophischen Zellen» gesprochen hatte ${ }^{13}$, hat er sich unter dem Eindruck von Jesionek und Kiolemenoglu zu der Ansicht bekannt, dass es sich um körperfremde Gebilde, und zwar von Protozoennatur, handle. Doch Ribbert konnte nicht entscheiden, ob ihnen irgendwelche pathogenetische Bedeutung zukam: «Es ist (...) bisher nicht möglich, über die Bedeutung der sehr wahrscheinlich als Protozoen aufzufassenden grossen Zellen etwas auszusagen ${ }^{14}$, und liess sie in seinem «Lehrbuch» unerwähnt ${ }^{15}$.

\section{Tietzes Mitteilung}

In einer 1905 veröffentlichten Arbeit hatte der damals am Breslauer Augustahospital als Chirurgieprofessor tätige Alexander Tietze von einem am 9. August 1898 stattgefundenen Eingriff berichtet, wobei eine als Lymphangiom diagnostizierte schmerzlose, daumengrosse Geschwulst der rechten Parotis eines sonst gesunden 4 Monate alten Knaben operativ entfernt wurde. Eine Woche nach der Operation konnte das Kind aus dem Krankenhaus entlassen werden, nach 8 Wochen war die Wundheilung abgeschlossen, jedoch bestand noch eine Lähmung der Fazialisäste derselben Seite. Bei der Nachuntersuchung ein halbes Jahr später gab es keine Drüsenschwellung mehr und keinen Rückfall, und es wurde ein erheblicher Rückgang der Fazialislähmung festgestellt ${ }^{16}$.

11 Jesionek und Kiolemenoglu, «Ueber einen Befund von protozoënartigen Gebilden in den Organen eines hereditär-luetischen Fötus». Münch. med. Wschr. 51/43, 1905-1907 (1904).

12 Ribbert (No. 1), S. 946.

13 Hugo Ribbert, «Über compensatorische Hypertrophie der Nieren». Arch. path. Anat. Physiol. 88/1, 11-27 (1882), S. 12.

14 Ribbert (No. 1), S. 948.

15 Hugo Ribbert, "Lehrbuch der allgemeinen Pathologie und der pathologischen Anatomie». F. C. W. Vogel Verlag, 6. Auflage, Leipzig, 1919.

16 Alexander Tietze, «Ein Protozoenbefund in einer erkrankten Parotis». Mitt. Grenz.g. Med. Chir. 14, 303-310 (1905), S. 305-306. 


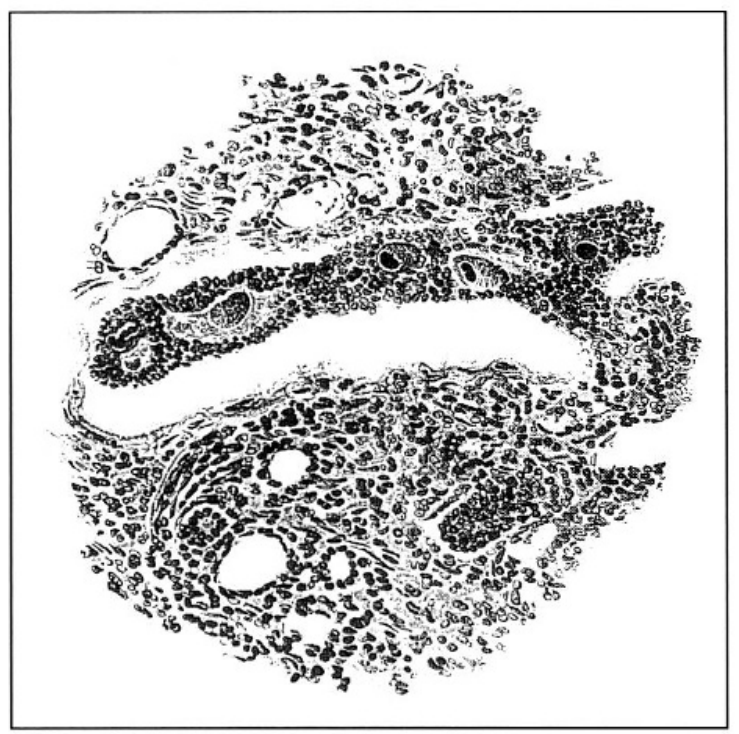

Abb. 1. Tietzes Darstellung von den als Amöben betrachteten Riesenzellen in einer der Drüsenschläuche der Parotis (Aus: Mitteilungen aus der Medizin und Chirurgie, Jahrgang 1905, Band XIV, Tafel VII, Gustav Fischer Verlag, Stuttgart/Jena/New York).

\section{Der pathologische Befund}

Die entfernte Geschwulst entpuppte sich als verändertes Parotisgewebe, ohne irgendein zusammengehöriges, fremdartiges Gebilde zu enthalten, das sich aus dem Gesamtbilde herausgehoben hätte. Die mikroskopische Untersuchung zeigte nebst einer stellenweise kavernomartigen Erweiterung sämtlicher Blutgefässe den Schwund der Drüsenelemente und den Ersatz derselben durch ein junges, zell- und kernreiches Bindegewebe, das in der Mehrzahl der Drüsenabschnitte zu einer festen Hepatisierung der Gewebe führte ${ }^{17}$. Als überraschenden, besonderen Befund hatte Tietze die Anwesenheit von eigentümlichen runden Gebilden in den Drüsengängen der Parotis hervorgehoben, die stark gequollenen Zellen glichen und die Grösse normaler Epithelzellen um ein 3-4faches übertrafen. $\mathrm{Ob}$ wandständig oder frei im Lumen liegend, diese Gebilde waren immer von einer dichten rundzelligen Infiltration umgeben (Abb. 1).

\section{Die Deutung der eigentïmlichen Gebilde}

Tietze war von Anfang an der Überzeugung, dass es sich bei diesen Gebilden um aus der Mundhöhle eingewanderte Protozoen handelte. Diese Ansicht wurde auch von den zu Rate gezogenen Zoologen Neisser und Flügge geteilt und schliesslich von Schaudinn bestätigt: «Es kann kein Zweifel sein,

17 Ibid., S. 306. 
dass es sich um Protozoen handelt, und zwar wahrscheinlich um Amöben, die der Entamoeba buccalis Prowazek am nächsten stehen könnten, etc.» ${ }^{18}$. Es war damals bekannt, dass die Mundhöhle nicht selten Aufenthaltsort gerade von solchen Parasiten sei und dass eine Einwanderung von pathogenen Zellen in die Parotis durch den Ductus Stenonianus erfolgen könne. Tietze vermutete, dass die Krankheitserscheinungen durch die massenhafte Überschwemmung der Drüse mit derartigen Elementen verursacht wurden. Dabei fiel ihm allerdings die Intaktheit der Parotisepithelien in der Umgebung der Protozoen auf, die dem Untergang der Drüsensubstanz in direkter Folge der Einwanderung der fremden Lebewesen widersprach.

\section{Die Auslegung der begleitenden Infiltration}

Tietze fand die Gebilde stets durch eine dichte rundzellige Infiltration begleitet, «welche sich gewissermassen wie eine Wolke in der Nachbarschaft niederlässt (...) und den feineren Gewebsaufbau zur vollkommenen Unkenntlichkeit verändert». Später wurde oft darüber nachgedacht, ob die protozoenartigen Zellen nicht gerade unter der Einwirkung dieser intensiven Entzündung entstandene entartete Körperzellen seien ${ }^{19}$. Doch es hatte sich schliesslich herausgestellt, dass die Infiltration stattdessen zur Entfernung der den Abwehrmechanismen als Fremdkörper imponierenden protozoenartigen Zellen dient. Ist diese Abwehrreaktion erfolgreich, so werden in den Geweben statt der entledigten protozoenähnlichen Gebilde nur noch die übriggebliebenen Infiltrationsherde aufgefunden. Es wurde ferner angedeutet, dass diese Reaktion das Auffinden zytomegaler Zellen erschweren, ja sogar verhindern, und damit eine positive histologische Diagnose vereiteln könnte ${ }^{20}$.

\section{Die nosologische Einordnung des Krankheitsbildes}

Tietze verglich den chronisch entzündlichen indurativen Prozess in der kindlichen Parotis mit den Beobachtungen des Breslauer Chirurgen Johann von Mikulicz-Radecki. Dieser hatte eine später nach ihm benannte Krankheit

18 Fritz Schaudinn, zit. in Tietze (No. 16), S. 304.

19 Ernest W. Goodpasture and Fritz B. Talbot, «Concerning the nature of ‘protozoan-like〉 cells in certain lesions of infancy». Amer. J. Dis. Child. 21/5, 415-425 (1921), S. 424.

20 James B. Arey, «Cytomegalic inclusion disease in infancy». Amer. J. Dis. Child. 88, 525-526 (1954), S. 526. 
beschrieben, die aus einer symmetrischen Schwellung von Tränen- und Mundspeicheldrüsen mit Untergang der spezifischen Drüsenelemente bestand und gewöhnlich vom Versiegen des Tränenflusses und Trockenheit des Mundes begleitet war $^{21}$. Dabei hatte v. Mikulicz schon in seinen ersten Publikationen die Frage erörtert, ob dies nicht aufgrund einer Noxe geschehen könne, welche von der Mundhöhle aus in die Ausführungsgänge der benachbarten Drüsen eindringt. Tietze wählte zur Veröffentlichung seines Berichtes die «Grenzgebiete der Medizin und Chirurgie» aus, da in dieser Zeitschrift schon mehrfach über die v. Mikulicz'sche Krankheit berichtet wurde.

\section{War Ribberts Abhandlung Tietze bereits bekannt?}

Tietze bemerkte: «die Angelegenheit verzögerte sich etwas», und zur Veröffentlichung seiner Beobachtungen kam es tatsächlich erst Jahre später, kurz nach der Publikation von Jesionek und Kiolemenoglus wie auch Ribberts Mitteilung. Dem Anschein nach hatten wederTietze noch Neisser, Flügge oder Schaudinn diese Mitteilungen wahrgenommen. Ob Tietze die Beschreibungen und Abbildungen der protozoenartigen Zellen bekannt waren, mag dahingestellt bleiben, doch es scheint nicht ausgeschlossen, dass ihn einer dieser Artikel zur Publikation seiner früher zur Seite gelegten Befunde bewog.

\section{Die Auswirkung von Tietzes Abhandlung}

Ausser einem Zitat seitens der amerikanischen Pathologen Smith und Weidman: «Tietze records a case of a parotid tumor (...) in which an amebiform organism was met .... ${ }^{22}$, blieb Tietzes Bericht in der Literatur ein halbes Jahrhundert lang unerwähnt. Da seine Abhandlung die erste klinisch-pathologische Beschreibung einer bis dahin unbekannten nosologischen Entität darstellte, ist es seltsam, dass sie von Zeitgenossen und Nachfolgern so lange übersehen wurde. Was Smith und Weidmans Bericht betrifft, so glaubten diese Autoren ebenfalls, dass Amöben, und zwar eine als Entamoeba mortinatalium bezeichnete neue Art, in den Organen von Totgeborenen zu identifizieren wären. Seltsamerweise waren den Amerikanern der Artikel von

21 Johann von Mikulicz, «Über eine eigenartige symmetrische Erkrankung der Thränen- und Mundspeicheldrüsen». Beitr. chir. Fortschr., Billroth Festschrift, 610-630 (1892).

22 Allen J. Smith and F. D. Weidman, «Infection of a stillborn infant by an amebiform protozoön (Entamoeba mortinatalium, N. S.)». Univ. Penn. med. Bull. 23, 285-298 (1910), S. 297. 
Jesionek und Kiolemenoglu sowie der von Ribbert anfangs auch nicht bekannt, doch sie veröffentlichten schon in der nächsten Nummer derselben Zeitschrift (University of Pennsylvania Medical Bulletin, Oktober, 1910) nachträglich zu ihrem Artikel einen Vermerk über diese Mitteilungen ${ }^{23}$.

\section{Die Virusätiologie}

Tietze wie Smith und Weidman und später auch Malowitschko und $\mathrm{Pu}-$ penko $^{24}$ betrachteten die protozoenartigen Gebilde als eine bis dahin unbekannte Amöbenart. Sie kamen demnach intuitiv darauf, dass es sich um eine Infektion, und zwar mit einem bis dahin unbekannten Erreger handelte. Die Entdeckung von Kerneinschlüssen in den Hautläsionen varizellenkranker Kinder ${ }^{25}$ und in febrilen Herpesbläschen ${ }^{26}$, welche den Kernveränderungen der protozoenartigen Zellen sehr ähnlich erschienen (in der deutschsprachigen Literatur später als Lipschütz Körperchen bekannt, in der angelsächsischen Literatur dagegen als «intranuclear inclusion body of Cowdry's type A» bezeichnet), hat den Verdacht bald auf eine Virusinfektion gerichtet ${ }^{27}$. Die bei Pocken, Trachoma, Tollwut usw. vorhandenen kleineren Plasmaeinschlusskörperchen wurden nämlich früher schon von Prowazek der Wirkung von Ultraviren zugeschrieben. Doch manche Pathologen verwarfen noch 1933 die Hypothese einer von Protozoen oder Viren herrührenden parasitären Genese und behaupteten weiterhin, dass die protozoenartigen Gebilde vielmehr entartete körpereigene Zellen, höchstwahrscheinlich einzellige Hamartome seien ${ }^{28}$. Erst die Züchtung des Speicheldrüsenvirus auf menschlichen embryonalen Fibroblastenkulturen hat die Virusätiologie dieser Zellveränderung unwiderlegbar und endgültig bewiesen ${ }^{29}$.

23 Allen J. Smith and F. D. Weidman, «Further note upon the occurrence of Entamoeba mortinatalium as a human parasite». Amer. J. trop. Dis. prev. Med. 2, 256-259 (1914), S. 256.

24 Elena Malowitschko und I. G. Pupenko, «Ein Fall von Amöbenansteckung der menschlichen Unterkieferdrüse». Arch. Schiffs. Tropenhyg. 38/1, 28-31 (1934), S. 31.

25 E. E. Tyzzer, «The histology of the skin lesions in varicella». Philippine J. Sci. (Manila) 1, 349-374 (1906).

26 Benjamin Lipschütz, «Untersuchungen über der Krankheiten der Herpesgruppe (Herpes zoster, Herpes genitalis, Herpes febrilis)». Arch. Dermat. Syph. 136/3, 428-482 (1921).

27 William C. Von Glahn and Alwin M. Pappenheimer, «Intranuclear inclusions in visceral disease». Amer. J. Path. 1/5, 445-465 (1925), S. 459 und 462.

28 Gian-Carlo Parenti, «Frequenza, morfologia ed interpretazione delle cosedette «cellule protozoosimili> nei tubuli escretori delle ghiandole salivari». Sperimentale 87, 157-189 (1933), S. $184-186$.

29 Margaret G. Smith, «Propagation in tissue cultures of cytopathogenic virus from human salivary gland virus disease». Proc. Soc. exp. Biol. Med. 92, 424-430 (1956). 


\section{Spätere kasuistische Berichte}

Obwohl Parenti ${ }^{30}$ und später Delvaux ${ }^{31}$ behaupteten, dass die auf die Speicheldrüsen begrenzte Anwesenheit zytomegaler Zellen ausschliesslich bei Kleinkindern und niemals bei Erwachsenen angetroffen werde, kam es in den nächsten Jahrzehnten wiederholt zur Veröffentlichung ähnlicher Fälle gerade bei Jugendlichen und Erwachsenen.

So fanden 1934 Malowitschko und Pupenko ${ }^{32}$ in den Ausführungsgängen der Unterkieferdrüse eines an den Folgen einer Knochensplitterverletzung der Speiseröhre verstorbenen Mannes rein zufällig «eine Menge Amöben». Die Gebilde lagen teils frei in den Höhlen der Ausführungsgänge, teils schienen sie die Zellen des Zylinderepithels auseinanderzuschieben und hindurchzuschlüpfen. Eine Zerstörung der Membrana propria oder das Eindringen der Amöben in das Zellparenchym war jedoch nicht zu erkennen. In der Drüse selbst liess sich ein von erweiterten Kapillaren und feinzelligem Infiltrat gekennzeichneter chronischer Entzündungsprozess nachweisen. Ihrem späten Verdacht, wonach in diesem Fall neben der Parotis möglicherweise auch die Speiseröhre und der Magen in Mitleidenschaft gezogen wurden, konnten die Autoren nicht nachgehen, da der Verstorbene inzwischen beerdigt worden war. Malowitschko und Pupenko glaubten, dass es sich um eine Verseuchung durch eine neue Amöbenart handle, die sie als Amoeba submaxillaris bezeichneten und der sie aufgrund der vorhandenen chronischen Entzündungserscheinungen pathogene Eigenschaften zuschrieben. Die ihrem Artikel beigefügten Mikrophotographien lassen die Gebilde jedoch zweifelsohne als zytomegale Zellen erkennen.

Danach veröffentlichte im Jahre 1960 Symmers $^{33}$ einen Fall von lokalisierter Zytomegalieerkrankung der Parotis bei einem 32jährigen, sonst gesunden Mann. Der seit 8 Jahren langsam heranwachsende Parotistumor stellte sich histologisch als typisches Adenolymphom heraus. Im Epithelium einiger Speichelgänge war die Anwesenheit zytomegaler Zellen unübersehbar, sie fehlten jedoch sowohl in anderen Teilen der Drüse als auch in der Geschwulst selbst. Sonstige Gewebsveränderungen wurden in der Mitteilung nicht erwähnt, und der Patient hatte nach seiner Heilung die Durchführung zusätzlicher Untersuchungen nicht abgewartet.

30 Parenti (No. 28), S. 184.

31 Thomas C. Delvaux, Jr., «Viral lesions complicating lymphoma in an adult. Localized cytomegalic inclusion disease, and a second viral infection». Amer. J. clin. Path. 28/3, 286-292 (1957), S. 286.

32 Malowitschko und Pupenko (No. 24), S. 31.

33 William St. C. Symmers, «Cytomegalic inclusion-body disease affecting the parotid gland of an adult».J. Path. Bact. 79, 406-408 (1960). 
Ferner haben Diosi und Rosiu ${ }^{34} 1965$ über eine bei einem 43jährigen sonst gesunden Mann aufgetretene und klinisch der Steinbildung verdächtigten Schwellung der linken Submaxillardrüse berichtet. In der beseitigten Geschwulst konnten mikroskopisch sowohl in den Drüsenazinen als auch in den Speichelgängen mehrere von einer starken interstitiellen lymphoretikulären Infiltration begleitete charakteristische zytomegale Zellen nachgewiesen werden. Kurz nach dem Eingriff, bevor weitere geplante Untersuchungen durchgeführt werden konnten, verliess der Patient das Krankenhaus geheilt.

1970 hat Linhartová ${ }^{35}$ den Fall eines 16jährigen schwarzen Mädchens veröffentlicht, bei dem eine langsam wachsende schmerzlose Geschwulst der linken Parotis festgestellt wurde. Die histologische Untersuchung des entfernten, den Tumor enthaltenden oberflächlichen Teiles der Drüse ergab, neben der Anwesenheit zytomegaler Zellen in einigen Drüsenläppchen, eine von ungewöhnlich intensiver lymphozytären Infiltration begleitete Atrophie der Azinen und der Speichelgänge. Dagegen konnte in anderen Drüsengängen die Rückbildung des Epithels beobachtet werden, wodurch es mehrschichtig wurde, ähnlich dem Epithel der Mundhöhle, von dem die Drüse abstammt. In der Literatur konnte Linhartová keinen Anhalt dafür finden, dass lymphoepitheliale Erkrankungen der Speicheldrüsen in irgendeinem Zusammenhang mit der Zytomegalie stehen würden, wies aber darauf hin, dass die Untersuchung weiterer ähnlicher Fälle diese Frage klären könnte.

In diesem Zusammenhang scheint auch Mietkes Artikel von $1958^{36}$ von Interesse, der über zytomegale Zellvorkommen in einem exzidierten Pseudotumor der Orbita berichtet. Mietke hatte diese Zellen als Hinweis auf das Vorliegen einer interstitiellen chronischen Entzündung der Tränendrüsen bzw. für den Endzustand einer solchen, d. h. Granulomabildung und Zirrhose, betrachtet.

Mittlerweile hatten der Hautarzt Gougerot $1925^{37}$, der Ophthalmologe Houwer $1927^{38}$ und der Jönköpinger Augenarzt Sjögren $1933^{39}$ ein dem rheu-

34 Peter Diosi and Natalia Rosiu, «Cytomegalic infection in the submaxillary glands of an adult». Path. Microbiol. (Basel) 28, 420-424 (1965).

35 Alena Linhartová, «Benign lymphoepithelial lesions of the parotid gland associated with cytomegaly». Amer. J. Dis. Child. 120, 562-563 (1970), S. 563.

36 Hans Mietke, «Über das Vorkommen zytomegaler Befunde in der Tränendrüse». Klin. Mbl. Augenheilkd. 127, 400-403 (1955).

37 Henri E. Gougerot, «Insuffisance progressive et atrophie des glands salivaires et muqueuses de la bouche, des conjonctives (et parfois des muqueuses, nasale, laryngée, vulvaire); 〈sécheresse〉 de la bouche, conjonctives, etc.». Bull. Soc. franç. Dermat. Syph. 32, 527-544 (1925).

38 A. W. Moulok Houwer, «De aetiologie der keratitis filamentosa». Ned. Tijdschr. Geneesk. 1, 1126-1132 (1927).

39 Hendrik S. C. Sjögren, «Zur Kenntnis der Keratoconjunctivitis sicca (Keratitis filiformis bei Hypofunktion der Tränendrüsen)». Acta Ophthal. Suppl. 2,1-151 (1933). 


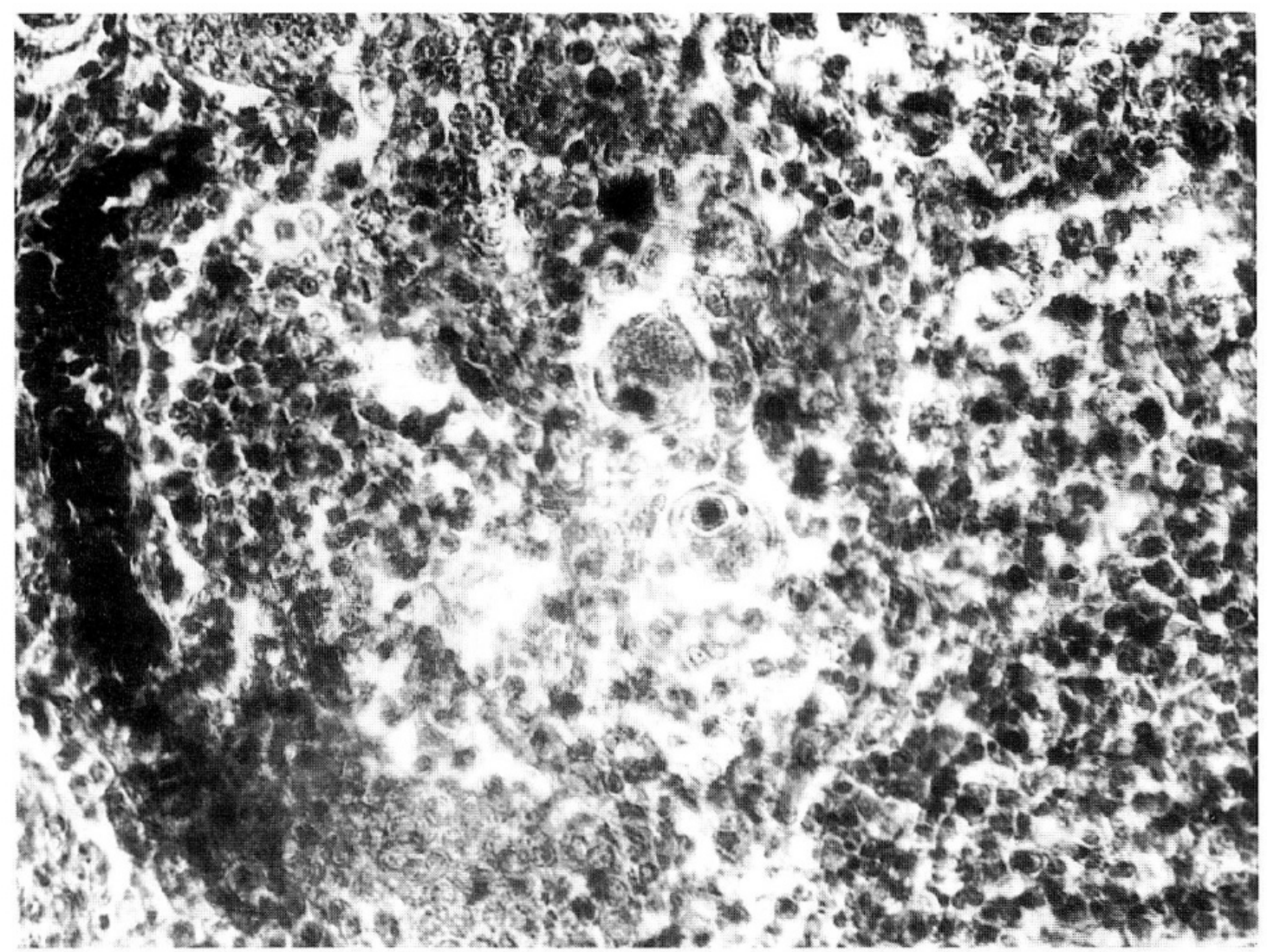

Abb. 2. Zwei zytomegale Zellen in der Bildmitte, von einem dichten Schwarm von Leukocyten umgeben (Mikrophotographie, aus der Kollektion des Verfassers).

matischen Formenkreis zugehöriges und mit der v. Mikulicz'schen Krankheit vergleichbares Syndrom unklarer Ursache beschrieben. Dieses war durch ungenügende Drüsenleistung, Trockenheit und Keratose der Schleimhäute (daher manchmal auch Sicca Syndrom - nach Leriche «Eil sec et bouche sèche» - oder auch Keratoconjunctivitis sicca genannt), Vergrösserung der Parotis mit Steinbildung und rheumatoide Polyarthritis gekennzeichnet und wurde seitdem als Gougerot-Houwers-Sjögren- oder Gougerot-SjögrenSyndrom und schliesslich als Sjögrens-Syndrom allgemein bekannt. Nach Einführung der serologischen Diagnose der Zytomegalie 1957 haben bei Patienten mit Sjögrens-Syndrom festgestellte positive Ergebnisse Aufsehen erregt $^{40,41}$ und zu Spekulationen geführt, wonach eine chronisch persistierende Infektion mit Zytomegalievirus als Ursache dieses Syndroms galt. Es

40 J. J. Thorn et al. «High levels of complement fixing antibodies against cytomegalovirus in patients with primary Sjögren's syndrome». Clin. exp. Rheumatol. 6/1, 71-74 (1988).

41 Edward J. Shilitoe, Troy E. Daniels, John P. Whitcher, Wibeke Strand, Norman Talal, and John S. Greenspan, «Antibody to cytomegalovirus in patients with Sjögren's syndrome, as determined by an enzyme linked immunosorbent assay». Arthritis Rheum. 25/3, 260-265 (1982). 
wurde nämlich angenommen, dass die durch die Zytomegalie-Virusinfektion freigelegten zellulären Antigene die körperliche Abwehr alarmierten und eine Autoimmunreaktion auslösten, welche dann zu den klinischen Erscheinungen dieses Syndroms führten ${ }^{42}$. Dazu sei erwähnt, dass einige Forscher auch die nach Knochenmarktransplantationen häufig auftretende und von der Anwesenheit zytomegaler Zellen begleitete interstitielle Pneumonie als Autoimmunkrankheit auffassen ${ }^{43}$.

\section{Gegenüberstellung der Fälle}

Die Aufzeichnungen einer Reihe ähnlicher Krankheitsfälle, bei denen die merkwürdigen Gebilde in den Gängen der Speicheldrüsen als zytomegale Zellen erkannt wurden (Abb. 2), deutet darauf hin, dass es sich wahrscheinlich auch in dem von Tietze veröffentlichten Fall um eine ZytomegalieVirusinfektion der Speicheldrüsen handelte. Diese Vermutung wird durch die offensichtliche Ähnlichkeit der in Abb. 1 dargestellten «Amöben» mit den in Abb. 2 abgebildeten zytomegalen Zellen bekräftigt. Das histologische Bild war in allen Fällen von einem chronisch-entzündlichen, indurativen Prozess und von der Erweiterung der Blutgefässe dominiert, wobei in den Gängen der Speicheldrüse die Anwesenheit der protozoenartigen Zellen durch eine dichte rundzellige Infiltration begleitet war, die zur ausgedehnten Zerstörung der Drüsenarchitektur führte. Klinisch hat sich das Auftreten eines langsam wachsenden, meist schmerzlosen, gutartigen Speicheldrüsentumors bei sonst gesunden Patienten, der chronische Ablauf und die rückfallose Heilung nach Entfernung des Tumors als kennzeichnend für dieses Krankheitsbild erwiesen. Gemeinsam war auch mehreren dieser Fälle die Diagnosestellung zu Lebzeiten des Patienten und die erfolgreiche chirurgische Behandlung. Diese Beobachtungsreihe zeigt, dass die als klinisch unbedeutsam geltende lokalisierte Zytomegalie-Virusinfektion der Speicheldrüsen in eine zwar seltene, doch klinisch manifeste subakute oder chronische Krankheit übergehen kann. Der auslösende Faktor, der die bis dahin latente Infektion aktiviert, die Rolle der Zytomegalie-Virusinfektion bei der Entstehung des Krankheitsbildes, ihre Beziehung zu den lymphoepithelialen Tumoren dieser Organe und zu der v. Mikulicz'schen Krankheit bzw. zum SjögrensSyndrom blieben jedoch ungeklärt.

42 J. C. Burns, «Persistent cytomegalovirus infection - The etiology of Sjögren's syndrome». Med. Hypothes. 10/4, 451-460 (1983).

43 J. A. Zaia, «The biology of human cytomegalovirus infection after bone marrow transplantation». Internat. J. Cell Cloning 4/Suppl. 1, 135-154 (1986), S. 146. 


\section{Tietzes Priorität}

Tietze war anscheinend der erste Kliniker, der mit der Zytomegalie-Viruskrankheit am Krankenbett konfrontiert wurde und über seine Beobachtungen ausführlich berichtet hatte. Sein früher Beitrag sichert ihm so eine unabstreitbare Priorität hinsichtlich der Beschreibung des klinisch-pathologischen Bildes der auf die Kopfspeicheldrüsen lokalisierten ZytomegalieErkrankung, der intra vitam erstellten Diagnose, der Erkennung protozoenartiger Gebilde in operativ entfernten Gewebefragmenten sowie der erfolgreichen chirurgischen Behandlung und nicht zuletzt der Genesung des Patienten. Zur Zeit seines Eingriffes 1898 lagen über die Krankheit mit protozoenartigen Gebilden noch keine Erkenntnisse vor. Zur ersten durch exfoliative Zytologie in vivo erstellten Diagnose ${ }^{44}$ und zur darauffolgend eingeführten operativen Behandlung der Zytomegalie-Viruskrankheit ${ }^{45} \mathrm{kam}$ es erst ein halbes Jahrhundert später.

44 George H. Fetterman, «A new laboratory aid in the clinical diagnosis of inclusion disease of infancy». Amer. J. clin. Path. 22, 424-425 (1952).

45 H. Stephen Gallagher, «Cytomegalic inclusion disease of infancy. Report of a case associated with cysts of the lung; with recovery following lobectomy». Amer. J. clin. Path. 22, 1147-1152 (1952). 